\title{
Neuroimaging as a Tool for Pain Diagnosis and Analgesic Development
}

\author{
Karolina Wartolowska and Irene Tracey \\ Centre for Functional Magnetic Resonance Imaging of the Brain, Department of Clinical Neurology, and Nuffield Department of \\ Anaesthetics, University of Oxford, Oxford, England, OX3 9DU, United Kingdom
}

Summary: Neuroimaging makes it possible to study pain processing beyond the peripheral nervous system, at the supraspinal level, in a safe, noninvasive way, without interfering with neurophysiological processes. In recent years, studies using brain imaging methods have contributed to our understanding of the mechanisms responsible for the development and main- tenance of chronic pain. Moreover, neuroimaging shows promising results for analgesic drug development and in characterizing different types of pain, bringing us closer to development of mechanism-based diagnoses and treatments for the chronic pain patient. Key Words: Neuroimaging, pain, analgesia, drug development.

\section{INTRODUCTION}

The pathophysiology of chronic pain conditions is not fully understood, which hinders development of new mechanism-based analgesic therapies. Unlike acute pain, there is no specific and effective medication to treat chronic pain. New drugs that appear on the market are mostly just refinements of existing drug classes.

Pain is not just a warning symptom informing our body of actual or potential damage to the tissue, although that is its primary function. Pain is also a complex, unpleasant sensation with sensory, emotional, and cognitive dimensions. In this article, we will mostly be concerned with chronic pain. The definition of chronic pain is pain that accompanies a chronic disease or has lasted longer than 3 months, despite resolution of the disease or healing of the injury that caused it. The important characteristic of chronic pain is that it loses its warning function and becomes a disorder in its own right, ${ }^{1}$ possibly even a disease. ${ }^{2}$

For the last 20 years, brain imaging methods, such as functional magnetic resonance imaging (fMRI) and positron emission tomography (PET), have greatly contributed to our understanding of the perception and modulation of the pain experience with several recent reviews covering

Address correspondence and reprint requests to: Karolina Wartolowska, M.D., Centre for Functional Magnetic Resonance Imaging of the Brain, Department of Clinical Neurology, and Nuffield Department of Anaesthetics, University of Oxford, Oxford, England, OX3 9DU, UK.E-mail: karolina@fmrib.ox.ac.uk; irene@fmrib.ox.ac.uk. these developments. ${ }^{3-7}$ It is now accepted that the central nervous system plays a crucial role in pain processing and many characteristics of chronic pain are caused by changes within the nervous system. ${ }^{6,8}$ Neuroimaging techniques provide a tool for understanding the mechanisms involved in generating and sustaining chronic pain. Moreover, neuroimaging can potentially be used as an objective, reliable, and perhaps more sensitive method to assess the efficacy of analgesic drugs, compared with subjective reporting, and as such, aid development of new treatment strategies. ${ }^{9-14}$

Most of our current knowledge of pain processing is based on acute pain or pain-model studies in healthy volunteers. These studies are easier to interpret as a standardized stimulus is used in a homogeneous population. From the brain imaging studies on experimental pain in healthy volunteers, we know that acute pain evokes a largely bilateral response in several brain regions, including the primary and secondary somatosensory, and the insular cortices, the anterior cingulate gyrus, the prefrontal cortex, and the thalamus. Pain-related activation in these regions can be amplified/attenuated with further activation identified in the posterior parietal cortex, brainstem, basal ganglia, amygdala, and cerebellum, as well as other areas dependent on the individual's mood, cognitive state, and context of the situation. ${ }^{3}$ From these studies, it is clear that there is no single "pain center" in the brain, but rather there is an extensive, interconnected network of cortical and subcortical struc- 
tures involved in the central processing and generation of a pain experience.

In short, pain is not a straightforward sensory process. First, the pain experience does not necessarily relate linearly to magnitude of the intensity of the nociceptive stimulus. As nociceptive information is transmitted along sensory pathways, it undergoes modulation, including both facilitation and inhibition mainly within the dorsal horn of the spinal cord. Second, pain is a multidimensional, unpleasant conscious experience strongly modulated by external factors (e.g., contextual) and internal factors (e.g., psychological, genetic). ${ }^{15-18}$ Neuroimaging studies have demonstrated that negative emotions, such as depression or anxiety, augment the perceived pain intensity, ${ }^{18}$ and increase the pain-related brain activation. ${ }^{19}$ How the pain is experienced is also affected by attention, ${ }^{20,21}$ anticipation, ${ }^{22}$ and pain memories. ${ }^{23}$ In addition, of course, the perception of pain changes in pathological states, such as inflammation or after lesion to the sensory nervous system. ${ }^{3,24}$

\section{EXPERIMENTAL AND CLINICAL PAIN: SAME OR DIFFERENT BRAIN REGIONS?}

To date, relatively few studies have focused on the neural correlates of experimental phasic pain in patients and even less on the ongoing, tonic pain. Therefore, it is not certain whether the same brain regions that are associated with the experimental pain are also involved in pathological chronic pain syndromes. ${ }^{25}$

In recent years, there have been an increasing number of clinical pain studies providing new insights into pathological mechanisms of chronic pain. The results of an early meta-analysis done by Apkarian et al. ${ }^{6}$ suggest that there are differences between pain processing in healthy volunteers versus chronic pain patients. The regions most frequently observed in connection with acute pain in healthy volunteers were the primary and secondary somatosensory cortices, the anterior cingulate cortex, the insular cortex, and the thalamus, whereas in chronic pain patients activation in these regions was reported less often (activated in $82 \%$ of healthy volunteers' studies vs $42 \%$ of patients' studies). The region most often reported activated in clinical pain studies was the prefrontal cortex $\left(81 \%\right.$ in patients vs $55 \%$ in healthy controls). ${ }^{6}$

The prefrontal cortex is involved in pain control mechanisms, in processing the ascending input from the spinothalamic tract, and it is a source of descending modulation via its connections to the brainstem's descending pain modulatory system. ${ }^{5}$ This region plays an important role in interoception, cognition, and processing of negative emotions and outcomes. ${ }^{7,26,27}$ Activation in the medial prefrontal cortex was reported during ongoing lower back pain, and was correlated with the intensity of the pain. ${ }^{28}$ The thalamus is another region affected by chronic pain. Several studies reported a decrease of cerebral blood flow and metabolic rate in the thalamus contralateral to the clinical pain. ${ }^{29-31}$ These changes normalize after successful pain treatment. ${ }^{32}$ Apkarian et al. ${ }^{6}$ suggest that patients have a decrease in the sensory aspect of pain processing and an increase in affective and cognitive processing of pain. This may be a result of clinical pain having a stronger emotional value. ${ }^{33} \mathrm{An}$ other possible explanation is that the ongoing pain leads to more generalized changes, affecting the baseline state, and leading to an altered response to evoked pain. ${ }^{6}$ This explanation is congruent with a study by Baliki et al. ${ }^{34}$ who reported changes in the default brain activity in chronic pain patients in comparison with healthy controls. This disruption of the default state network may explain the different activation pattern observed in clinical pain studies than in healthy controls, as well as the cognitive and behavioral impairment reported in chronic pain patients. ${ }^{35}$

Pain is not passively transmitted to the brain. The nociceptive inputs are changed and modulated at each level of the pain neuraxis. The inputs from the dorsal horn of the spinal cord are modulated (inhibited or facilitated) by the endogenous descending pain modulatory system. This system involves monoaminergic projections from the higher brain regions, including the prefrontal cortex, the anterior cingulate cortex, the insular cortex, the amygdala, the hypothalamus, and the brainstem. It has been demonstrated, in human models of pain, that the pain modulatory system is critical for sustaining central sensitization in menthol, ${ }^{36}$ and capsaicin-evoked allodynia. ${ }^{37,38}$ In a study on capsaicin-evoked allodynia and its modulation by gabapentin, the brainstem was the main region where the effect of the drug on sensitization was observed. ${ }^{38}$ The pain modulatory system is also crucial for sensitization and maintaining chronic pain in patients. ${ }^{39}$ Patients with chronic pain, either neuropathic or inflammatory, have altered endogenous control of pain with increased descending facilitation or impaired inhibition. $^{40-42}$

From the previous description, it is clear that several potential brain regions and networks might serve as objective, noninvasive "biomarkers" for specific aspects of the pain experience, and as such, might be used to aid diagnosis and to be used as surrogates for determining analgesic efficacy.

\section{EVOKED VERSUS SPONTANEOUS PAIN IN CHRONIC PAIN PATIENTS}

In chronic pain patients, there are differences in the brain activation pattern in response to evoked pain (i.e., acute pain) in comparison with the ongoing, tonic pain. The evoked pain results in similar brain activation as the acute pain in healthy controls. ${ }^{43-45}$ Tonic clinical pain 
results in a different brain activity pattern than evoked clinical pain; for example, dynamic mechanical allodynia in patients with post-herpetic neuralgia results in different activation patterns than the ongoing pain. ${ }^{43,46}$ Also the evoked pain differs from the ongoing, tonic pain in patients with arthritis. ${ }^{44}$ This observation is very important for treatment development, as it highlights the fact that the evoked pain typically used in pain studies, even though it is disease-related, is not the same as the ongoing pain the patients experience during the course of their disease, and often describe as their most problematic symptom.

In a study on chronic back pain it was shown that ongoing, tonic pain correlates with stronger activation in the regions involved in emotion, cognition, and motivational drive, such as the medial prefrontal cortex, the rostral anterior cingulate cortex, as well as the thalamus and amygdala. ${ }^{28}$ However, the increase of the intensity of their clinical pain correlates with activation in the regions observed during acute pain processing (i.e., the somatosensory cortices and the insular cortex). ${ }^{28}$ Kulkarni et al. ${ }^{44}$ also observed differences between ongoing arthritic pain and evoked pain. Both conditions activated several brain regions, sensory, affective, and motivational, but the tonic pain was associated with activation in the brainstem and greater activation in the regions involved in affective processing of pain. The long-term treatment effect and reduction of tonic pain correlates with activation in the areas processing emotions and reward. ${ }^{46}$

\section{ARE WE CLOSER TOWARD USING IMAGING FOR A MECHANISM-BASED CLASSIFICATION OF CHRONIC PAIN?}

Different types of allodynia can result in distinct brain activation patterns, which is congruent with the results of psychophysical studies and the hypothesis that different types of allodynia have different pathophysiological mechanisms. In a study on syringomyelia by Ducreux et al., ${ }^{47}$ the authors reported different results of psychophysical tests, as well as distinct activation patterns between patients with cold allodynia and patients with tactile allodynia. The only region that was consistently activated during both types of allodynia in this study was the pre-frontal cortex. Cold allodynia evokes responses in dorsolateral prefrontal cortex and brainstem (regions usually involved in sensitization), in addition to the regions activated in response to the noxious cold. ${ }^{48}$ Activation in the prefrontal cortex was found also by Schweinhardt et al. ${ }^{49}$ in their study on neuropathic pain patients, as well as in other studies on capsaicin-evoked allodynia in healthy volunteers. ${ }^{50}$

As with many preclinical models of disease, there are always limitations regarding how well they mirror the patient condition. This is certainly the case for preclinical pain models. Despite displaying key symptoms of chronic pain, patients are difficult to assess behaviorally regarding the presence and severity of these symptoms, in particular the affective components. Furthermore, models usually reflect a single mechanism or symptom of pain, whereas in chronic pain conditions there is usually more than one symptom and mechanism driving the pain. ${ }^{51}$ Likely these shortcomings have contributed to the low predictive capacity of analgesic efficacy in patients of these preclinical models of pain. Neuroimaging, by itself, or when coupled to standardized psychophysical assessments such as quantitative sensory testing, may help to diagnose which mechanism is responsible or dominant for pain in a particular patient. ${ }^{52}$ For many years it has been known that there is a need for better characterization and mechanism-based classification of pain. ${ }^{53}$

\section{NEUROIMAGING AS A TOOL FOR ASSESSING DRUG-INDUCED ANALGESIA AND "REVERSE-TRANSLATION" TO PRECLINICAL MODELS}

Currently, to be able to measure pain we have to rely on a patient's subjective report using uni-dimensional rating scales. These scales, although easy to use and accepted in clinical and research settings, are imprecise, relative, context-dependent, and vary significantly between and within patients. Moreover, pain scales do not provide information of the underlying pain mechanisms. ${ }^{54}$ Neuroimaging offers an objective and quantitative method to assess pain by measuring the magnitude of pain-related brain activation. ${ }^{55}$ This method has its limitations, but it is a step forward from using the patients' report.

Arguably, the same case can be made for animal models of chronic pain where the behavioral read-out is even more difficult to relate to underlying mechanisms. There is now increasing enthusiasm to apply functional imaging methods in preclinical models of pain in combination with drugs. ${ }^{12,54,56,57}$ The body of knowledge we have gleaned from human studies regarding central pain mechanisms could be more aggressively "reverse translated" to guide these preclinical models. We believe this to be a promising future direction and potential opportunity for neuroimaging methods in the next decade.

As previously described, neuroimaging is a promising tool to study sustaining mechanisms and exacerbating chronic pain. It is still poorly understood how chronic pain is maintained and why it persists. At the same time, treatment depends on mechanisms rather than cause. ${ }^{58}$ Understanding the neurophysiological mechanisms of pain would lead to optimization of therapy, help in better identifying patients who would respond 
to treatment, and potentially help in identifying new treatment strategies.

Neuroimaging may be used as a tool to assess the effects of peripherally and centrally acting analgesics. Brain imaging studies are able to demonstrate effects of drugs on the central nervous system comparable with the behavioral measures. There are several studies published on remifentanil, a rapidly acting opioid receptor agonist, and its effect on pain processing. Functional MRI has proven to be sensitive enough to detect changes in brain activity with increased concentration of the drug, and to demonstrate the correlation between the brain activation and both drug dose and pain ratings. ${ }^{59-62}$ Functional neuroimaging can be also used to study the pharmacodynamics and pharmacokinetics of analgesic drugs. ${ }^{63}$ Furthermore, imaging is a tool that can improve or clarify our understanding of the likely mechanism of action of approved analgesic drugs, as well as their side effect profiles, such as the study on the capsaicin-evoked sensitization model and gabapentin by Iannetti et al. ${ }^{38}$ Moreover, and as previously mentioned, neuroimaging is useful for translation and reverse translation between preclinical models, healthy volunteer models, and patients, ${ }^{64}$ as illustrated in FIG. 1. Furthermore, brain imaging techniques may be used in drug development as a measure of drug efficacy with assessment of plasma drug concentration as a measure of exposure. This would provide an objective marker of treatment effect in phases I and II of drug development, therefore improving and accelerating the drug discovery process (FIG. 1).

There are, however, certain limitations in the application of functional imaging methods in clinical studies. First, the disease or medication may affect the neurophysiological process we are measuring as a surrogate marker of brain activity (e.g., changes in neurovascular coupling or changes in metabolic activity). Therefore, it is important to control for these effects while designing drug studies using neuroimaging methods. There are several ways of dealing with these limitations, such as using control task to assess global hemodynamic effects, inde- pendently assessing the baseline physiological state, e.g., by using arterial spin labeling methods, or by measurements of physiological parameters. Second, none of the functional imaging methods have both superior temporal and spatial resolution. For example, using multi-modal imaging, by combining fMRI with electroencephalography or with magnetoencephalography, allows us to establish in which order brain regions become active in response to a painful stimulus. Combining fMRI with PET makes it feasible to study neurochemical changes such as decrease in opioid binding that normalizes after reduction of pain. ${ }^{65}$ Recently, it has become possible to collect whole brain data using arterial spin labeling rather than just a single slice. ${ }^{66}$ This opens up new possibilities (e.g., controlling for baseline blood flow in fMRI studies, comparing global changes before and after treatment, or studying ongoing, continuous pain). This method allows quantitation of the blood flow, not just relative changes as measured using fMRI.

Clearly there are many other, nonpharmacological ways of producing analgesia (e.g., surgical, cognitive behavioral therapy, alternative). Only a few imaging studies have attempted to determine the neural basis for any analgesia produced, ${ }^{67-69}$ but again these methods lend themselves to further study and improvements in our understanding of these additional lines of therapy. Understanding the neural basis of pleasure and relief provides alternative strategies and potential targets for intervention aimed at taking the pain away. ${ }^{70}$

\section{CONSEQUENCES OF NOT ALLEVIATING CHRONIC PAIN: STRUCTURAL BRAIN CHANGES}

The results of neuroimaging studies on brain structure or chemistry demonstrate that chronic pain affects not only the function of the brain, but can lead to long-lasting changes. Some of these changes seem to be reversible, when the pain is alleviated. ${ }^{8}$ However, some studies suggest that the pain-related changes may reflect neuro-
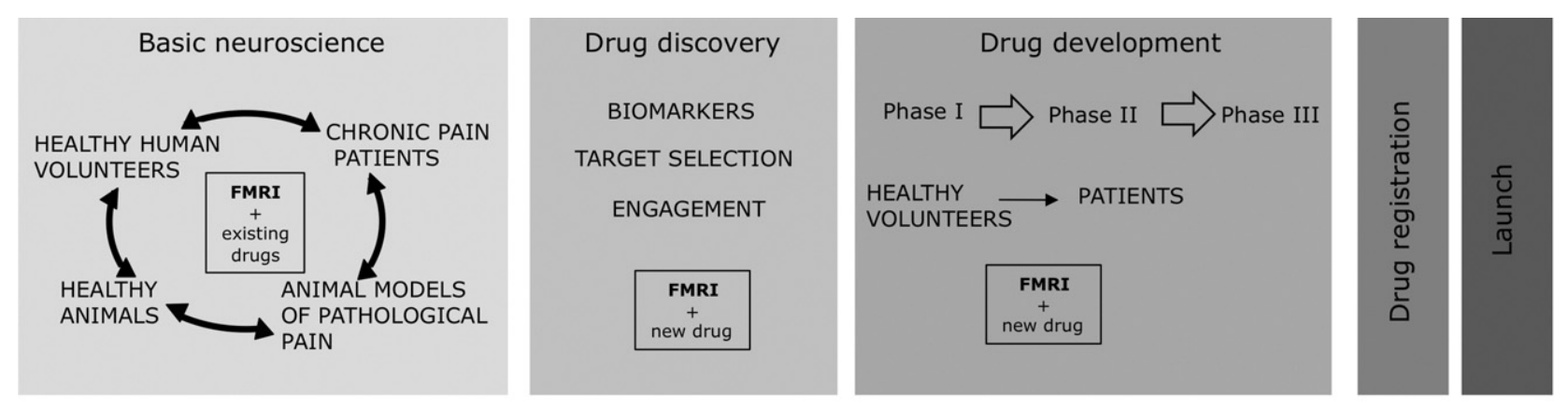

FIG. 1. Graphical illustration of where neuroimaging methods, such as FMRI, could possibly fit in the drug discovery process across disease areas, including pain. 
degeneration, rather than neuronal reorganization. ${ }^{71-73}$ The changes were observed in several brain areas and may be an acceleration of age-related brain atrophy. ${ }^{74}$ Apkarian et al. ${ }^{71}$ demonstrated that in patients suffering from lower back pain, the density of the grey matter decreases in several cortical and subcortical areas, including the prefrontal cortex. There was also a decrease of the $\mathrm{N}$-acetylaspartate concentration (i.e., a marker of neuronal well-being) in the prefrontal cortex of these patients. $^{75}$ The reduction of grey matter has been described not only in lower back pain, ${ }^{16,76}$ but also in several other chronic pain conditions such as migraine, ${ }^{77,78}$ chronic tension headache,${ }^{72}$ irritable bowel syndrome, ${ }^{79}$ and fibromyalgia. ${ }^{74}$ It remains to be determined whether these changes are due to chronic pain conditions itself, the drugs the patients are taking, the lifestyle changes due to disuse or a combination of these factors. Current work attempts to disentangle the causal nature of this degeneration. ${ }^{2}$

\section{CONCLUSIONS}

Neuroimaging is a noninvasive method that objectifies pain and allows the neurophysiology of pain processing, as well as the pathological changes that occur in chronic pain conditions, to be studied. There is a need to better understand the mechanisms responsible for the generation and maintenance of chronic pain and for the development of new, effective therapies. In a relatively short time period, neuroimaging studies have contributed significantly to this goal and our understanding of the pathophysiology of chronic pain. We firmly believe that neuroimaging-based methods may potentially improve mechanism-based classification of pain and lead to better diagnostic accuracy and identification of patients who would respond to treatment. Neuroimaging may improve drug development by making the evaluation of treatment efficacy easier and more objective, and critically, by identifying new therapeutic targets. Therefore, we believe that neuroimaging methods will become part of the pharmaceutical industry's analgesic development process, as illustrated in FIG. 1, but that they will rapidly penetrate additional areas of application in the coming decade.

\section{REFERENCES}

1. Loeser JD, Treede RD. The Kyoto protocol of IASP Basic Pain Terminology. Pain 2008;137:473-477.

2. Tracey I, Bushnell C. How neuroimaging studies have challenged us to rethink: is chronic pain a disease? J Pain 2009;(in press).

3. Tracey I, Mantyh PW. The cerebral signature for pain perception and its modulation. Neuron 2007;55:377-391.

4. Bingel U, Tracey I. Imaging CNS modulation of pain in humans. Physiology 2008;23:371-380.

5. Wiech K, Ploner M, Tracey I. Neurocognitive aspects of pain perception. Trends Cogn Sci 2008;12:306-313.
6. Apkarian AV, Bushnell MC, Treede RD, Zubieta JK. Human brain mechanisms of pain perception and regulation in health and disease. Eur J Pain 2005;9:463-484.

7. Wiech K, Tracey I. The influence of negative emotions on pain: Behavioral effects and neural mechanisms. Neuroimage 2009;47: 987-994.

8. Maihofner C, Handwerker HO, Neundorfer B, Birklein F. Cortical reorganization during recovery from complex regional pain syndrome. Neurology 2004;63:693-701.

9. Wise RG, Tracey I. The role of fMRI in drug discovery. J Magn Reson Imaging 2006;23:862-876.

10. Schweinhardt P, Bountra C, Tracey I. Pharmacological FMRI in the development of new analgesic compounds. NMR Biomed 2006;19:702-711.

11. Borsook D, Bleakman D, Hargreaves R, Upadhyay J, Schmidt KF, Becerra L. A 'BOLD' experiment in defining the utility of fMRI in drug development. Neuroimage 2008;42:461-466.

12. Borsook D, Becerra L, Hargreaves R. A role for fMRI in optimizing CNS drug development. Nat Rev Drug Discov 2006;5:411424.

13. Borsook D, Becerra L. Functional imaging of pain and analgesia-a valid diagnostic tool? Pain 2005;117:247-250.

14. Matthews PM, Honey GD, Bullmore ET. Applications of fMRI in translational medicine and clinical practice. Nat Rev Neurosci 2006;7:732-744.

15. Keefe FJ, Rumble ME, Scipio CD, Giordano LA, Perri LM. Psychological aspects of persistent pain: current state of the science. $\mathrm{J}$ Pain 2004;5:195-211.

16. Schmidt-Wilcke T, Leinisch E, Ganbauer S, et al. Affective components and intensity of pain correlate with structural differences in gray matter in chronic back pain patients. Pain 2006;125:89-97.

17. Seminowicz DA, Davis KD. Cortical responses to pain in healthy individuals depends on pain catastrophizing. Pain 2006;120:297306.

18. Craig A. Pain, inflammation and a nodule after IV medication. Adv Nurse Pract 2005;13:21-22.

19. Giesecke T, Gracely RH, Grant MA, et al. Evidence of augmented central pain processing in idiopathic chronic low back pain. Arthritis Rheum 2004;50:613-623.

20. Tracey I, Ploghaus A, Gati JS, et al. Imaging attentional modulation of pain in the periaqueductal gray in humans. J Neurosci 2002;22:2748-2752.

21. Eccleston C, Crombez G. Attention and pain: merging behavioral and neuroscience investigations. Pain 2005;113:7-8.

22. Fairhurst M, Wiech K, Dunckley P, Tracey I. Anticipatory brainstem activity predicts neural processing of pain in humans. Pain 2007;128:101-110.

23. Gedney JJ, Logan H. Memory for stress-associated acute pain. J Pain 2004;5:83-91.

24. Bingel U, Schoell E, Buchel C. Imaging pain modulation in health and disease. Curr Opin Neurol 2007;20:424-431.

25. Moisset X, Bouhassira D. Brain imaging of neuropathic pain Neuroimage 2007;37(suppl 1):S80-88.

26. Dolan RJ. Emotion, cognition, and behavior. Science 2002;298: 1191-1194.

27. Rushworth MF, Kennerley SW, Walton ME. Cognitive neuroscience: resolving conflict in and over the medial frontal cortex. Curr Biol 2005;15:R54-56.

28. Baliki MN, Chialvo DR, Geha PY, et al. Chronic pain and the emotional brain: specific brain activity associated with spontaneous fluctuations of intensity of chronic back pain. J Neurosci 2006;26:12165-12173.

29. Hsieh JC, Belfrage M, Stone-Elander S, Hansson P, Ingvar M. Central representation of chronic ongoing neuropathic pain studied by positron emission tomography. Pain 1995;63:225-236.

30. Pagni CA, Canavero S. Functional thalamic depression in a case of reversible central pain due to a spinal intramedullary cyst. Case report. J Neurosurg 1995;83:163-165.

31. Kupers RC, Gybels JM, Gjedde A. Positron emission tomography study of a chronic pain patient successfully treated with somatosensory thalamic stimulation. Pain 2000;87:295-302.

32. Di Piero V, Jones AK, Iannotti F, et al. Chronic pain: a PET study 
of the central effects of percutaneous high cervical cordotomy. Pain 1991;46:9-12.

33. Price DD. Psychological and neural mechanisms of the affective dimension of pain. Science 2000;288:1769-1772.

34. Baliki MN, Geha PY, Apkarian AV, Chialvo DR. Beyond feeling: chronic pain hurts the brain, disrupting the default-mode network dynamics. J Neurosci 2008;28:1398-1403.

35. Apkarian AV, Sosa Y, Krauss BR, et al. Chronic pain patients are impaired on an emotional decision-making task. Pain 2004;108: 129-136.

36. Seifert F, Maihöfner C. Representation of cold allodynia in the human brain - a functional MRI study. Neuroimage 2007;35: $1168-1180$.

37. Zambreanu L, Wise RG, Brooks JC, Iannetti GD, Tracey I. A role for the brainstem in central sensitisation in humans. Evidence from functional magnetic resonance imaging. Pain 2005;114:397-407.

38. Iannetti GD, Zambreanu L, Wise RG, et al. Pharmacological modulation of pain-related brain activity during normal and central sensitization states in humans. Proc Natl Acad Sci U S A 2005; 102:18195-18200.

39. Suzuki R, Rygh LJ, Dickenson AH. Bad news from the brain: descending 5-HT pathways that control spinal pain processing. Trends in Pharmacological Sciences 2004;25:613-617.

40. Ren K, Dubner R. Descending modulation in persistent pain: an update. Pain 2002;100:1-6.

41. Gebhart GF. Descending modulation of pain. Neurosci Biobehav Rev 2004;27:729-737.

42. Gwilym SE, Keltner JR, Warnaby CE, et al. Psychophysical and functional imaging evidence supporting the presence of central sensitization in a cohort of osteoarthritis patients. Arthritis Rheum 2009; (in press).

43. Geha PY, Baliki MN, Wang X, Harden RN, Paice JA, Apkarian AV. Brain dynamics for perception of tactile allodynia (touchinduced pain) in postherpetic neuralgia. Pain 2008;138:641-656.

44. Kulkarni B, Bentley DE, Elliott R, et al. Arthritic pain is processed in brain areas concerned with emotions and fear. Arthritis Rheum 2007;56:1345-1354.

45. Baliki M, Katz J, Chialvo DR, Apkarian AV. Single subject pharmacological-MRI (phMRI) study: modulation of brain activity of psoriatic arthritis pain by cyclooxygenase-2 inhibitor. Mol Pain 2005; $1: 32$.

46. Geha PY, Baliki MN, Chialvo DR, Harden RN, Paice JA, Apkarian AV. Brain activity for spontaneous pain of postherpetic neuralgia and its modulation by lidocaine patch therapy. Pain 2007; 128:88-100.

47. Ducreux D, Attal N, Parker F, Bouhassira D. Mechanisms of central neuropathic pain: a combined psychophysical and fMRI study in syringomyelia. Brain 2006;129:963-976.

48. Seifert F, Maihofner C. Representation of cold allodynia in the human brain - a functional MRI study. Neuroimage 2007;35: $1168-1180$.

49. Schweinhardt P, Glynn C, Brooks J, et al. An fMRI study of cerebral processing of brush-evoked allodynia in neuropathic pain patients. Neuroimage 2006;32:256-265.

50. Lorenz J, Cross D, Minoshima S, Morrow T, Paulson P, Casey K. A unique representation of heat allodynia in the human brain. Neuron 2002;35:383-393.

51. Klein T, Magerl W, Rolke R, Treede RD. Human surrogate models of neuropathic pain. Pain 2005;115:227-233.

52. Cruccu G, Anand P, Attal N, et al. EFNS guidelines on neuropathic pain assessment. Eur J Neurol 2004;11:153-162.

53. Woolf CJ, Bennett GJ, Doherty M, et al. Towards a mechanismbased classification of pain? Pain 1998;77:227-229.

54. Chizh BA, Greenspan JD, Casey KL, Nemenov MI, Treede RD. Identifying biological markers of activity in human nociceptive pathways to facilitate analgesic drug development. Pain 2008;140: 249-253.

55. Coghill RC, McHaffie JG, Yen YF. Neural correlates of interindividual differences in the subjective experience of pain. Proc Natl Acad Sci U S A 2003;100:8538-8542.

56. Jones KL, Finn DP, Governo RJ, et al. Identification of discrete sites of action of chronic treatment with desipramine in a model of neuropathic pain. Neuropharmacology 2009;56:405-413.
57. Guasti L, Richardson D, Jhaveri M, et al. Minocycline treatment inhibits microglial activation and alters spinal levels of endocannabinoids in a rat model of neuropathic pain. Mol Pain 2009;5:35.

58. Sindrup SH, Jensen TS. Efficacy of pharmacological treatments of neuropathic pain: an update and effect related to mechanism of drug action. Pain 1999;8:389-400.

59. Wagner KJ, Sprenger T, Kochs EF, Tolle TR, Valet M, Willoch F. Imaging human cerebral pain modulation by dose-dependent opioid analgesia: a positron emission tomography activation study using remifentanil. Anesthesiology 2007;106:548-556.

60. Wise RG, Rogers R, Painter D, et al. Combining fMRI with a pharmacokinetic model to determine which brain areas activated by painful stimulation are specifically modulated by remifentanil. Neuroimage 2002;16:999-1014.

61. Tracey I. Prospects for human pharmacological functional magnetic resonance imaging (phMRI). J Clin Pharmacol 2001;(suppl): 21S-28S.

62. Shyu BC, Kiritsy-Roy JA, Morrow TJ, Casey KL. Neurophysiological, pharmacological and behavioral evidence for medial thalamic mediation of cocaine-induced dopaminergic analgesia. Brain Res 1992;572:216-223.

63. Wise RG, Williams P, Tracey I. Using fMRI to quantify the time dependence of remifentanil analgesia in the human brain. Neuropsychopharmacology 2004;29:626-635.

64. Borsook D, Becerra LR. Breaking down the barriers: fMRI applications in pain, analgesia and analgesics. Mol Pain 2006;2:30.

65. Jones AK, Cunningham VJ, Ha-Kawa S, et al. Changes in central opioid receptor binding in relation to inflammation and pain in patients with rheumatoid arthritis. Br J Rheumatol 1994;33:909_ 916.

66. MacIntosh BJ, Pattinson KT, Gallichan D, et al. Measuring the effects of remifentanil on cerebral blood flow and arterial arrival time using 3D GRASE MRI with pulsed arterial spin labelling. J Cereb Blood Flow Metab 2008;28:1514-1522.

67. Owen SL, Green AL, Stein JF, Aziz TZ. Deep brain stimulation for the alleviation of post-stroke neuropathic pain. Pain 2006;120: 202-206.

68. Dhond RP, Kettner N, Napadow V. Neuroimaging acupuncture effects in the human brain. J Altern Complement Med 2007;13: 603-616.

69. Derbyshire SW, Whalley MG, Stenger VA, Oakley DA. Cerebral activation during hypnotically induced and imagined pain. Neuroimage 2004;23:392-401.

70. Leknes S, Tracey I. A common neurobiology for pain and pleasure. Nat Rev Neurosci 2008;9:314-320.

71. Apkarian AV, Sosa Y, Sonty S, et al. Chronic back pain is associated with decreased prefrontal and thalamic gray matter density. J Neurosci 2004;24:10410-10415.

72. Schmidt-Wilcke T, Leinisch E, Straube A, et al. Gray matter decrease in patients with chronic tension type headache. Neurology 2005;65:1483-1486.

73. Grachev ID, Fredrickson BE, Apkarian AV. Abnormal brain chemistry in chronic back pain: an in vivo proton magnetic resonance spectroscopy study. Pain 2000;89:7-18.

74. Kuchinad A, Schweinhardt P, Seminowicz DA, Wood PB, Chizh BA, Bushnell MC. Accelerated brain gray matter loss in fibromyalgia patients: premature aging of the brain? J Neurosci 2007;27: 4004-4007.

75. Grachev ID, Fredrickson BE, Apkarian AV. Brain chemistry reflects dual states of pain and anxiety in chronic low back pain. J Neural Transm 2002;109:1309-1334.

76. Buckalew N, Haut MW, Morrow L, Weiner D. Chronic pain is associated with brain volume loss in older adults: Preliminary evidence. Pain Medicine 2008;9:240-248.

77. Schmidt-Wilcke T, Ganssbauer S, Neuner T, Bogdahn U, May A. Subtle grey matter changes between migraine patients and healthy controls. Cephalalgia 2008;28:1-4.

78. Rocca MA, Ceccarelli A, Falini A, et al. Brain gray matter changes in migraine patients with T2-visible lesions: a 3-T MRI study. Stroke 2006;37:1765-1770.

79. Davis KD, Pope G, Chen J, Kwan CL, Crawley AP, Diamant NE. Cortical thinning in IBS: implications for homeostatic, attention, and pain processing. Neurology 2008;70:153-154. 